\title{
A benchmark strategy for the experimental measurement of contact fabric
}

\author{
Max Wiebicke · Edward Andò • Václav Šmilauer • Ivo Herle • \\ Gioacchino Viggiani
}

Received: date / Accepted: date

\begin{abstract}
The mechanics of granular materials can be better understood by experimental measurement of fabric and its evolution under load. X-ray tomography is a tool that is increasingly used to acquire threedimensional images and thus, enables such measurements.
\end{abstract}

Our previous study on the metrology of interparticle contacts revealed that the most common approaches either fail to accurately measure contact fabric or introduce a strong bias. Methods to improve these measurements (i.e., the detection and orientation of contacts) were proposed and validated.

This work develops a strategy to benchmark image analysis tools that can be used for the determination of contact fabric from tomographic images. The discrete element method is used to create and load a reference specimen for which the fabric and its evolution is precisely known. Chosen states of this synthetic specimen are turned into realistic images taking into account inherent image properties, such as the partial volume effect, blur and noise. The application of the image analysis tools on these images validates the findings of the metrological study and highlights the importance of addressing the identified shortcomings, i.e., the sys-

Max Wiebicke and Ivo Herle

Institute of Geotechnical Engineering, Technische Universität

Dresden, D-01062 Dresden, Germany

Tel.: +49-351-46332313

Fax: +49-351-46334131

E-mail: max.wiebicke@tu-dresden.com

Edward Andò and Gioacchino Viggiani

Univ. Grenoble Alpes, CNRS, Grenoble INP, 3SR, F-38000

Grenoble, France

Václav Šmilauer

woodem.eu, Prague, Czech Republic tematical over-detection of contacts and the strong bias of orientations when using common watersheds.

Keywords image analysis · fabric · interparticle contacts $\cdot$ DEM $\cdot$ x-ray CT

\section{Introduction}

Historically, soils are most commonly treated in a continuum framework in either theoretical modeling or the interpretation of experimental results. For most of the time, soil mechanicians were only able to observe and measure soil behaviour on the boundaries of a studied specimen. It was impossible to look into a specimen while loading it. It was acknowledged, however, that the micro-mechanics, i.e., the behaviour at the grainscale, drives the overall response of any granular material. Model granular materials, e.g., coins, provided first insights [1]. In these and following experiments, two-dimensional (planar) grains were loaded and photographs were acquired at specific stages of the loading [2]. Using photogrammetry [3], insight into the micromechanical behaviour was gained for either $2 \mathrm{D}$ model materials or granular materials in specific loading conditions that allowed photos to be acquired, e.g., in a biaxial loading apparatus.

Experiments on real granular materials were restricted to post-mortem analysis $[4,5]$ : similar, yet not identical, specimens were loaded to specific stages and subsequently frozen in this state, e.g., using epoxy resin. This allowed for thin cuts to be taken and analysed under a microscope.

On the simulation front, the development of the discrete element method (DEM) has allowed micro-mechanical investigations of the particle-scale behaviour of granular materials [6]. In these simulations, the response of 
a granular material is mainly controlled by the contact models which describe the mechanical interactions between particles. Applications of the discrete element method targeting soil behaviour have produced numerous valuable results and a better understanding of the micro-mechanisms linked to the overall behaviour $[7$, 8]. Despite continuously improving contact models and ever evolving DEM frameworks (e.g., [9]), DEM remains a numerical tool that needs further experimental validation. Its contact models are phenomenological, grain shapes are restricted (for an exception, see e.g., [9]) and surface properties of natural materials cannot be directly modeled.

With the rise of x-ray computed tomography (CT) in the last decade(s), full-field measurements of specimens finally became possible [10]. Specific set-ups in x-ray CT chambers allow for an acquisition of tomographies during a macroscopic loading of the specimen [11]. Different methods have been used and improved to measure the kinematics of the granular materials during loading, such as the digital image correlation [12, $11,13]$ or an individual particle tracking approach [14]. These experiments and tools allowed new insights into the mechanics at the grain-scale, in particular in the formation of shear bands $[10,15,13]$. Only recently, attempts to measure the granular fabric and its evolution at specific loading stages have been conducted [16, $17,18]$. There are several inherent features in x-ray tomographic images, that influence the analysis of such images, e.g., the detection of contacts and the determination of their orientation. Regarding the measurement of fabric the most problematic and apparent of these inherent features are blur, noise and the partial volume effect (PVE) [19,20]. Despite its significance, a quantitative analysis of the accuracy for the tools that are used to determine the fabric, i.e., contact normal orientations, principal particle orientations or void orientations, has not been undertaken until recently [21]. This study on the metrology of individual contacts focused only on two particles being in contact instead of big granular assemblies. The accuracy of the contact detection as well as the determination of their orientation were quantitatively demonstrated. Advanced techniques to improve the measurements were proposed.

The aim of this contribution is to extend the findings of [21] on individual contacts to a granular assembly that evolves under a macroscopic loading in order to assess what amplitude of fabric changes can be measured with realistic granular rearrangements. One main concern is the detection of small fabric changes that can occur e.g., upon unloading and reloading cycles. As these measurements and their quantification are difficult on real experiments e.g., using x-ray tomography, we start to study the problem from synthetic, but realistic $^{1}$ images. The advantage of these images is the controllability of the inherent features as well as the perfect knowledge of the fabric. For this purpose, the discrete element method is used to create and load a numerical, mechanically representative specimen. Realistic images of these DEM specimens are then created using Kalisphera [22] which generates analytically-correct 3D images of spheres.

Sphere packings are the object of many studies on the description of granular media [23]. One of the most studied properties is the structure of these packings, e.g., a random loose packing and a jamming transition [24]. Random packings have been studied from frictionless to frictional spheres, monodisperse to polydisperse particle sizes under compression and shear to predict these structural measures $[25,26]$. We decided to limit our analysis to the most common contact fabric measures for natural granular materials in geomechanics, which mostly deals with frictional, polydisperse and irregularly shaped grains: the coordination number and the fabric tensor.

The main object of this work is to present a benchmark test for the extraction of contact fabric from tomographies of granular materials. Our approach is somehow similar to [27], where DEM simulation were conducted based on tomographic data from sphere packings. Their aim, however, was the investigation of the mechanics in contrast to ours which is the accuracy of the fabric evaluation from tomographic images. Although we employ sphere packings, the methodology is not restricted to spherical, but aimed at more general shapes. Thus, the image analysis tools never assume a particular particle shape to support the extraction of information from the tomographies. Another similar approach was used to determine constriction sizes of pores from DEM simulations [28]. Binary images were created from the numerical sphere packings and processed by watershed segmentation. The results were compared with the constriction sizes determined directly on the DEM specimen.

All image-based calculations are done with the opensource Software spam (The Software for the Practical Analysis of Materials) [29]. The scripts to reproduce the results presented in this work are available in an open repository [30].

\footnotetext{
1 In this case realistic images are images that have similar properties as X-ray tomographies.
} 


\section{Contact-based measurements}

In the following section, the image analysis steps that are necessary to extract the contact fabric from x-ray tomographies are presented and the challenges of such analyses are highlighted. A simplified workflow of a general image analysis is presented in Figure 1.

The first step of producing an x-ray CT is the acquisition of radiographies of the specimen at various angles. These acquired radiographies serve as the basis for the reconstruction of a three-dimensional grey-scale image, the tomography.

In order to determine contact orientations, contacts have to be detected first. Therefore, the grey-scale image is transformed into a binary image where only two phases are distinguished - solid and void. This can be achieved using a variety of methods (global, local, adaptive thresholds, etc.) as described in [31]. The most common and easiest method is to apply a global threshold, which attributes all grey values up to a certain threshold to either one of the two phases. The calibration can be based on physical quantities (such as the actual volume of the solid phase if known) or image statistics (such as Otsu's threshold [32]). The next essential step in identifying the individual grains is the segmentation of the binary image. The most common segmentation techniques (in experimental geomechanics) are watershed algorithms, e.g., a standard topological watershed [33] or advanced watersheds, such as the random walker [34]. Once segmented, the image can then be labelled by assigning a unique number to each individual grain. Based on the segmented and labelled image, the contacts between apparently touching particles can be detected and the contact planes can be fitted (e.g., via principal component analysis) through the contact area. The contact orientation can be identified as the normal to that plane.

The accuracy of the crucial steps is analysed in [21]. The main findings are that the standard image analysis tools, briefly described above, are not sufficient to produce reliable results and that these techniques have either to be adapted or replaced by more advanced methods to detect contacts and determine their orientation as reliable as possible.

\subsection{Major results of the study on the metrology}

To methodically study the accuracy of the contact detection and orientation, the investigation started with analysing synthetic images of spheres, where particle shape and image properties such as blur and noise can be controlled, and followed up with high resolution to- mographies of manufactured spheres (to control the shape) and a natural granular material (Hostun sand).

The main source of uncertainty in contact detection is the binarisation of the grey-scale image and, particularly, the PVE [20]. The approach that was chosen to determine the accuracy of contact detection is described in detail in [21]. Two particles, that are initially in contact, were incrementally translated from each other to check up to which distance they still appear to be in contact in the image. The results of the analysis with the most common approach (the global threshold) are shown in Figure 2 with solid lines. A perfect detection would appear as 1 at 0 distance and zero for distances $>0$, i.e., with a probability of finding contacts of one at zero distance and zero at higher distances. An obvious over-detection can be observed in Figure 2. The main reason for the systematical over-detection of contacts is the PVE in regions where two particles are close to each other. In order to minimise the over-detection, a local thresholding is proposed: contacts that are detected with the global threshold are checked again with a higher, local threshold that is applied directly on the grey-values in the apparent contact region. It is found that this approach works well for rounded particles, less for more angular particles, e.g., Hostun sand grains. The results of chosen local thresholds are plotted in Figure 2 in dotted lines.

The same images, i.e., synthetic images of spheres as well as high resolution images of manufactured spheres and Hostun sand grains, were taken for the study on the accuracy of the determination of contact orientations. The contact orientations were determined by fitting a contact plane on the contact zone using principal component analysis. The two different watershed methods mentioned above, a topological watershed and the random walker [34] (as implemented in [35]), were used for the segmentation of the binary image. The topological watershed results in a labelled image in which the contact region can be identified from the labels in direct contact. The random walker assigns a probability to each voxel of either belonging to one or the other particle in contact which allows to determine the contact surface from the probability field on a subvoxel basis, whereas the former method can only work on the voxel basis. The error is defined as the angle between a reference and the determined orientation.

Spheres were chosen for this and the former analysis because the reference contact orientation is known from the branch vector, that is either imposed in the synthetic images or precisely measured from the center of mass of the two apparently touching particles [36]. The reference orientation of the contacts in Hostun sand was defined as the orientation that can be determined at the 


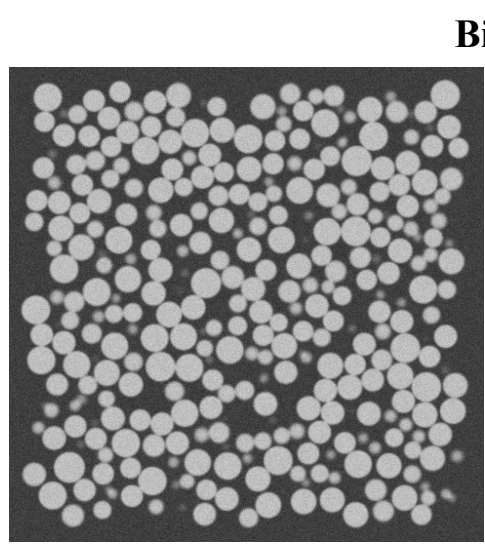

Grey-scale image
Binarisation

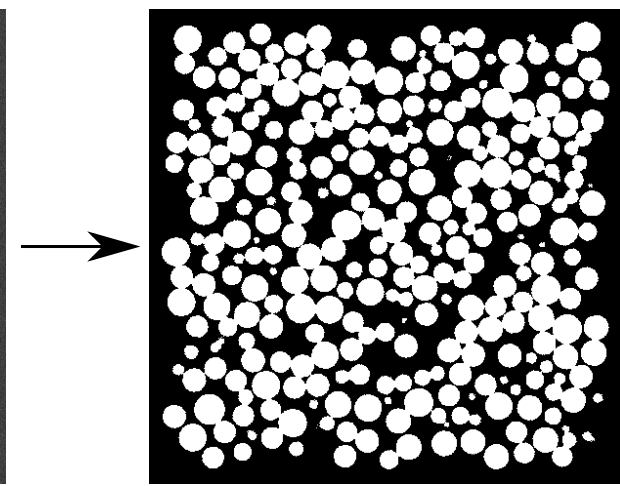

Binary image
Segmentation and labelling

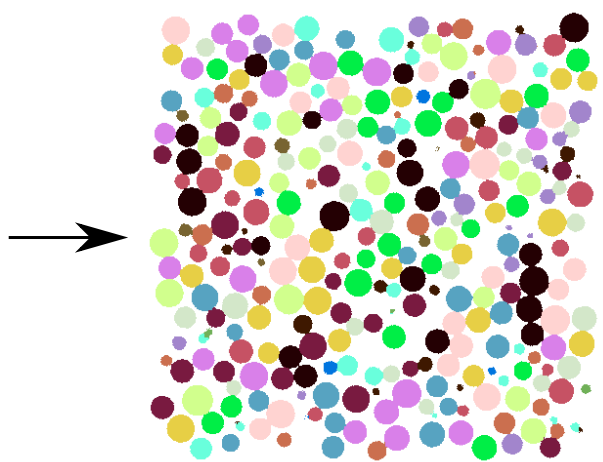

Labelled image

Fig. 1 Simplified sketch of the image analysis steps - from a grey-scale image (left) to a binary image (centre) to a segmented and labelled image (right).

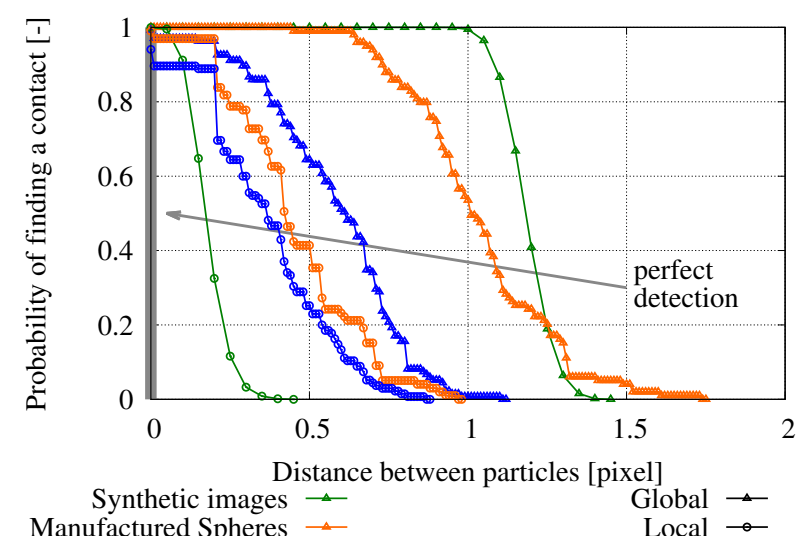

Manufactured Spheres $\triangleq$

Hostun Sand $\rightarrow$

Fig. 2 Results of the contact detection analysis from [21]

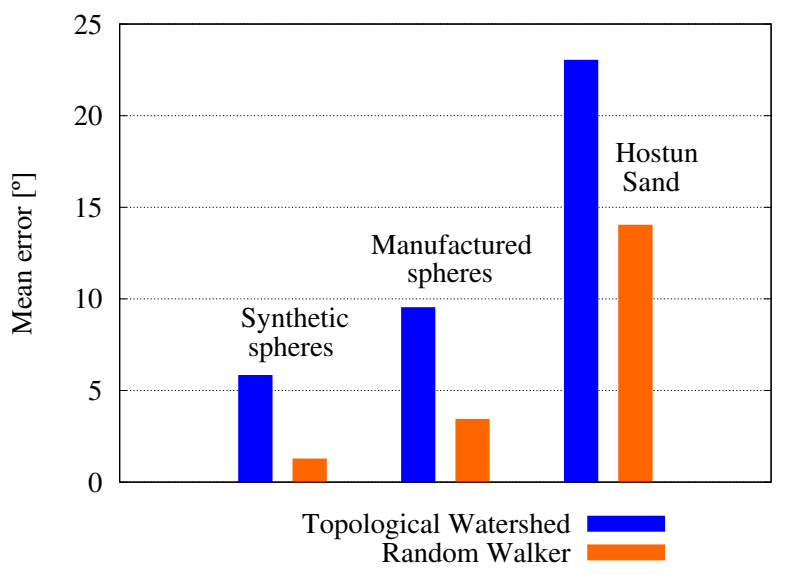

Fig. 3 Mean error of contact orientation for a diameter of 27 pixel - from the analysis in [21] smallest pixel size $(1 \mu \mathrm{m})^{2}$. The results of this part of the study are shown in Figure 3 for a relative resolution $d_{50} /$ pixel size of 27 pixel. The results are displayed for all materials in the analysis: the synthetic images of spheres, the high resolution tomograhies of manufactured spheres and the high resolution tomographies of Hostun sand. It can be concluded that the random walker yields more accurate orientations for all materials tested when the focus lies on individual contacts, i.e., only two particles touching each other.

\subsection{Image analysis tools}

All the image analysis tools mentioned above that were either tested or proposed to overcome problems in the description of inter-particle contacts were included in the open source analysis software spam [29]. It has evolved to cover needs of data analysis from 3D x-ray tomography work and correlated random fields with mechanical applications. The contact detection and orientation tools from [21] are included in the label toolkit of spam.

The complete image analysis that is applied to obtain the results in this work is summarised in minimal python scripts using spam. In order to enable the reproduction of this image analysis, these scripts are available online in a free repository [30]. The source data from the discrete element simulations (positions and radii of the spheres at every step of the analysis) can

\footnotetext{
2 Note: Due to the angular and non-convex shape of the Hostun sand grains, the description of the orientation however is not objective, as highlighted in [21]: when observing the same grains in different spatial resolutions, contacts might artificially be created (due to the PVE) or multiple contacts points at which two grains are touching might be merged to appear as a single contact.
} 
be found in the repository as well, and should be easy to obtain with any 3D DEM code.

\section{Testing and validating the measurement tools}

The study on the metrology focused completely on the detection and orientation of individual contacts, i.e., only two particles being in contact. In this work we develop a benchmark test in order to assess whether the image analysis tools work on images of assemblies of thousands of particles and how accurate the results are. In order to assess the accuracy, the reference, i.e., the true fabric, has to be known. The discrete element method is used to create assemblies of spherical particles and to obtain particle displacements due to externally imposed mechanical loads. Realistic grey-scale images of these numerical assemblies at different loading stages are then created using Kalisphera [22] in the same way as in [21] for individual particles in contact.

It has to be noted that due to the spherical shape of the particles branch vectors could be used to precisely determine the contact orientations. We employ the chosen image analysis approach because it has to be extendible to round (not necessarily spherical) particle shapes for which the equality of branch vector and contact orientation does not hold in general. The accuracy of the determination of the contact orientations of round and spherical particles, however, should be similar as the study on the metrology showed that the accuracy follows the contact topology.

As mentioned in the introduction, there exist various methods to detect contacts. Three different methods of estimating contact numbers were compared in [37] on monodisperse sphere packings. The best results were obtained using a contact number scaling function presented in [38], which however, is assuming spherical particles shapes. This approach was extended to packings of ellipsoids in [39] and [40]. As our research is generally dealing with irregular shapes and polydisperse packings, our approaches cannot profit from the mentioned results.

\subsection{DEM simulations as a reference}

The open source, discrete element software WooDEM [41] is used to create the numerical specimen and to load it mechanically. In order to directly consider the findings from [21], the particles are chosen to have a spherical shape. The particle size distribution (PSD) of the specimen is linear and ranges from $(3.2$ to 4.8$) \cdot 10^{-4} \mathrm{~m}$ and is thus in the range of the materials that were investigated in [21] and that will be used in real experiments in the $\mu$-CT: manufactured spheres with $d=4 \cdot 10^{-4} \mathrm{~m}$ and Hostun sand with $d_{50}=3.38 \cdot 10^{-4} \mathrm{~m}$. Periodic boundary conditions and a standard triaxial loading (axisymmetric) with a final unloading step are chosen for the simulation. The simulation itself is only needed to create mechanically meaningful states of the granular assembly for the subsequent image analysis and not to probe the mechanics. Thus, a simple, linear (Cundall) contact model [6] is considered as sufficient. The parameters of the contact model are chosen to be: $E=50 \cdot 10^{6} \mathrm{kPa}, k_{t} / k_{n}=0.4$ and $\tan \varphi=0.4$ with $E$ being Young's modulus, $k_{n}, k_{t}$ the normal and tangent stiffness respectively and $\varphi$ the friction angle. The initial package of 5522 spheres based on the given PSD is created and then isotropically compressed to $50 \mathrm{kPa}$. At pre-defined axial strains the fabric of the assembly, i.e., the position of the particles and the contact orientations, is extracted to determine the evolution of the fabric upon loading. The macroscopic response, i.e., the stress-strain response calculated at the boundaries of the specimen, is plotted in Figure 4.

\subsection{Turning DEM specimen into images}

The chosen, exported fabric states are turned into realistic grey-scale images using Kalisphera [22]. The pixel size, that is needed to convert the DEM data (positions and radii of spheres given in $[\mathrm{m}]$ ) to actual, pixelised data for the the image (given in [pixel]), is set to $15 \cdot 10^{-6} \mathrm{~m}$, which corresponds to a common setting of a tomography of a triaxial experiment on sand (e.g., [14]). A 32-bit floating point image of each sphere is created, where the values 0.0 and 1.0 correspond to a pure void and a pure solid voxel, respectively. The individual images are then subsequently added to an image of the big assembly. In some cases the overlap of particles on contacts leads to values higher than 1.0, which are flattened to 1.0. In order to apply blur and noise the values of the image are compressed to lie in the range of $(0.25 ; 0.75)$ corresponding to void and solid voxels, respectively. A Gaussian blur operator is applied to the image and random noise based on a Gaussian distribution is applied onto the blurred image. Representative levels of blur and noise for the $\mu$-CT in Laboratoire 3SR in Grenoble were calibrated on chosen images in [21]: the level of blur was found to be $\approx 0.8$ and the standard deviation of the Gaussian distribution for random noise was chosen to 0.03 . The final 32-bit floating point images are turned to 8-bit integer images (i.e., values between 0 and 255), because the image analysis on real tomographic images is usually carried out on this data type to save memory. The whole procedure is summarised in Figure 5. 

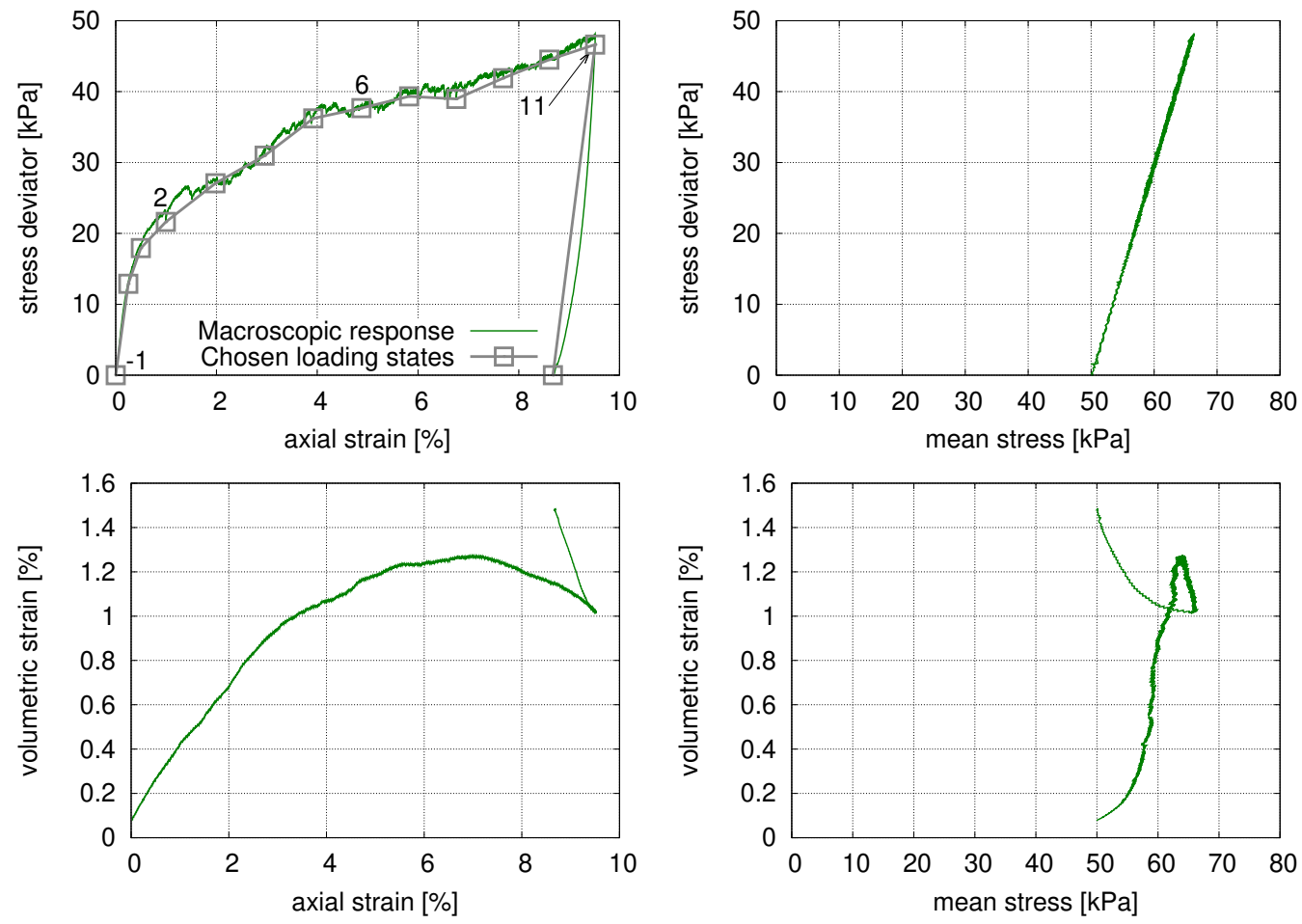

Fig. 4 Macroscopic response of a triaxial specimen from the discrete element simulation. The chosen loading states for the creation of the synthetic images are indicated in the upper left figure with grey boxes. Four states are given by their ID to reference them specifically in the analysis.

Woo(DEM)

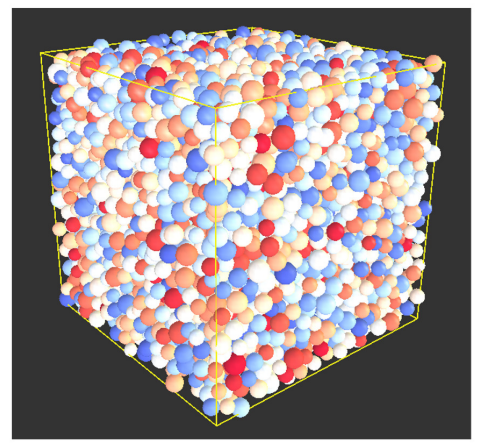

3D rendering of the image

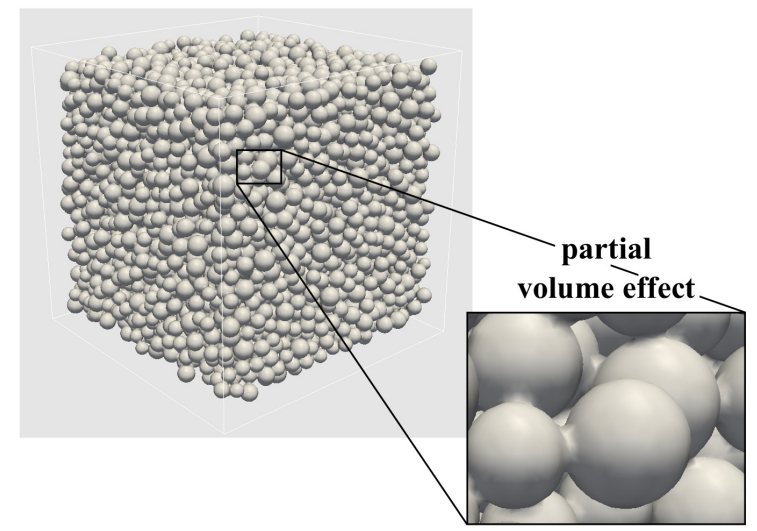

\section{Slices through the 3D image}

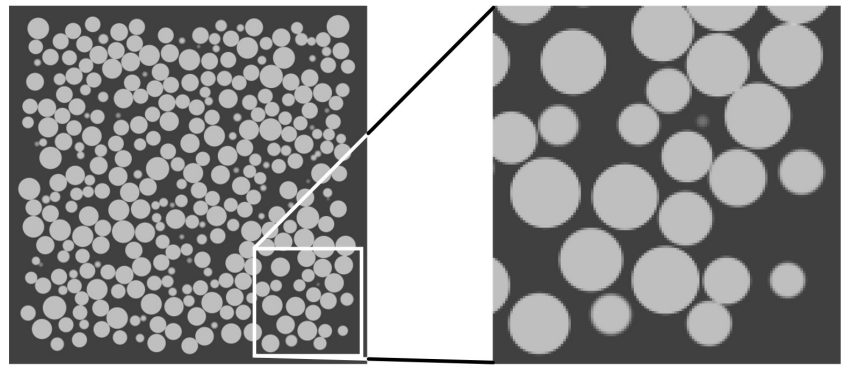

perfect image
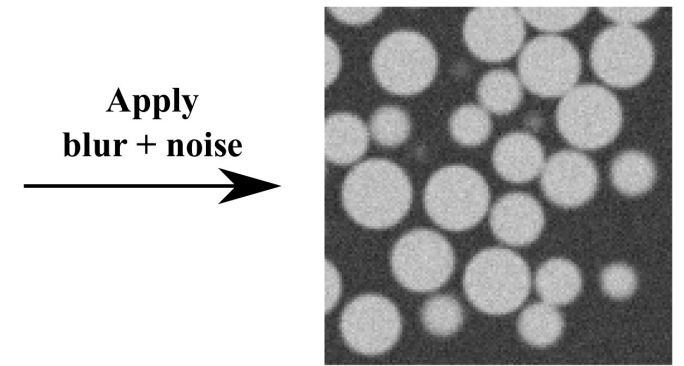

realistic image

Fig. 5 Illustration of how a synthetic specimen created with the discrete element method is turned into a realistic image. 


\subsection{Analysis of the reference and computed fabric}

The advanced image analysis techniques, that were described above and in more detail in [21], are applied to the resulting images. In order to reduce the overdetection of contacts, a local threshold of 180 (8bit unsigned integer images $)^{3}$ is applied to the globally detected contacts. A ITK inter-pixel watershed as implemented in [29] is applied to the binary image for the initial segmentation and labelling. The contact orientations are determined using a principal component analysis on the contacting voxels in the initially labelled image. Furthermore, the random walker [34] is then employed on contacting particles for the determination of the contact orientations from the resulting probability map.

A scalar value describing the fabric of the granular assembly is the coordination number, i.e., the number of contacts per particle. The average coordination number $C_{n}$ is given by:

$C_{n}=\frac{2 \cdot C}{N_{p}}$

with $C$ and and $N_{p}$ being the number of contacts and the number of particles, respectively. The coordination number is directly determined from the amount of detected contacts and should thus reflect the findings from [21], namely the systematic over-detection of contacts. The evolution of the average coordination number with the macroscopic loading is plotted in Figure 6. As to be expected, the number of contacts from the images is dramatically over-detected using the standard global approach with a mean deviation of $50 \%$. The local refinement of the detected contacts leads to a coordination number that is close to the reference from the simulation with a mean deviation of $3 \%$. Even though a local refinement is employed, some contacts are still over-detected. This is because the local threshold was chosen in order not to loose real contacts, see the dotted orange line in Figure 2 and thus, some apparent contacts are always over-detected.

Contact orientations can be plotted in different ways. Herein, we choose Lambert azimuthal equal area projections for the representation of orientations. In order to use this projection, each orientation (vector) is flipped such that it has a positive vertical axis. A simplified sketch of how these orientations are plotted and binned (when considering thousands of orientations) is shown in Figure 7.

The resulting plots of orientations at different, significant stages of the loading are shown in Figure 8:

3 This value corresponds to 0.7 for 32 bit floating point images, which was found to yield acceptable results in [21].

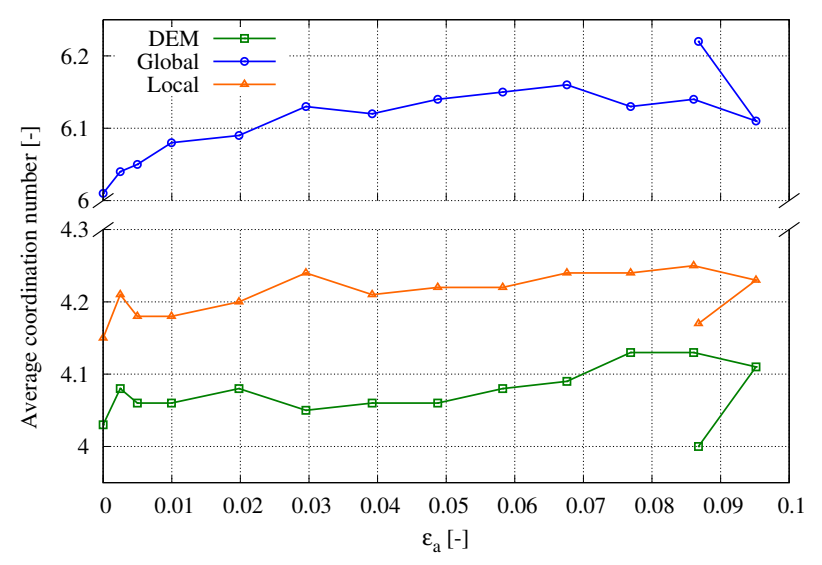

Fig. 6 Plot of the evolution of the average coordination number with the macroscopic loading. Comparison of the reference from the simulation and the results of the global and local contact detection of the image.

the reference orientations, that are directly exported from the DEM simulation are given in the upper row. The middle row shows the orientations that are calculated directly on the initial segmentation by ITK. The lower row depicts the orientations that are determined from the random walker segmentation of two contacting particles. In order to use similar scales for every loading state and to facilitate the comparison, the plots are normalised with the average number of orientations per bin.

The distribution of contact orientations in the DEM simulation (reference) evolve from a rather random configuration in the initial and the pre-stress peak stage to a clear vertically aligned contact fabric with ongoing loading.

The orientations from the initial segmentation and labelling do not resemble the reference distribution. As found in $[42,43]$, orientations that are calculated on the basis of pixel watersheds are strongly biased towards favourable orientations. In this specific case of the ITK watershed the orientations tend towards $\approx 45^{\circ}$ with respect to the Cartesian axes. This was found to be the reason for the high error in the determination of contact orientations [21], see Figure 3.

The contact orientations determined based on the random walker segmentation agree with the reference distribution. The reason for the better agreement is the probability map from the random walker, which allows interpolating the contact zone within the pixel instead of following pixel boundaries.

To quantitatively describe the errors of the image analysis approaches, the individual orientations must be related to their corresponding reference from the DEM simulation. As each contact is identified by the 

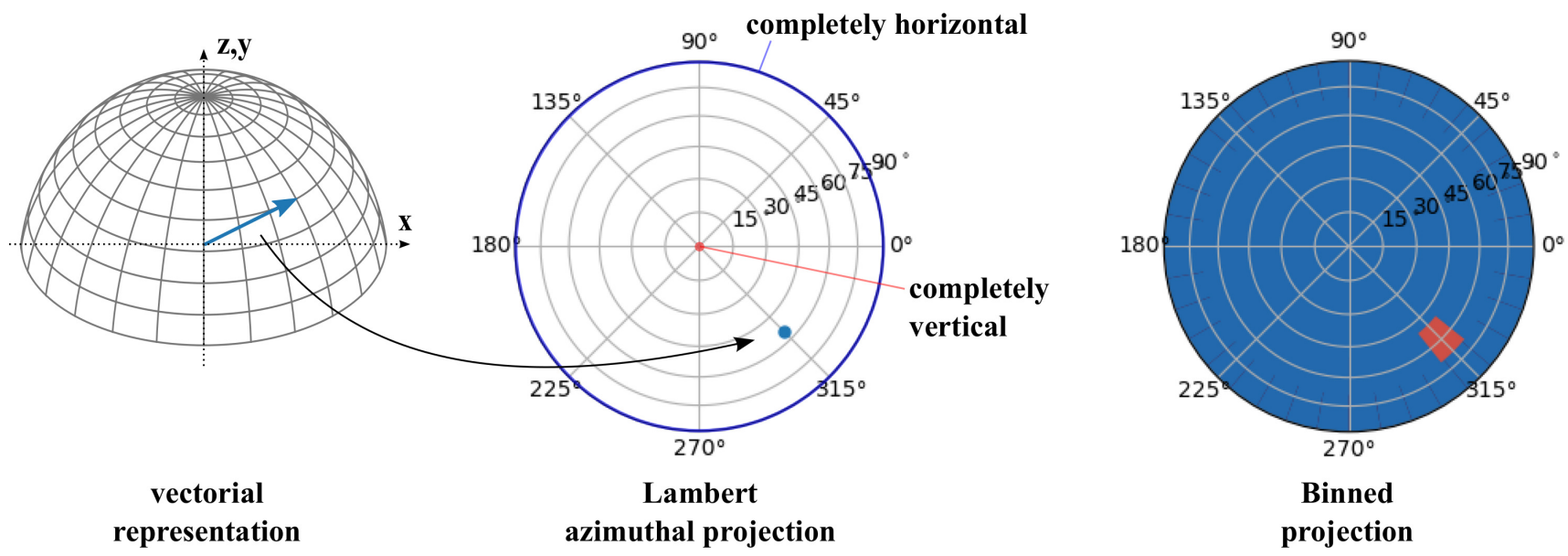

Fig. 7 Visualisation of the plotting of individual orientations and the binning to simplify plots of many orientations. A Lambert azimuthal equal area projection is used in this work.

labels of the contacting spheres and the labels in the DEM simulations are not equivalent to the labels in the images, a topographic mapping from DEM to image is established. The error of each orientation is determined by computing the angle between the orientation measured in the image and its corresponding reference in the simulation. It has to be noted that not all orientations could be compared due to the problematic detection of contacts in the image which was described before and plotted in Figure 6. A histogram of the errors of all orientations in state 2 (as highlighted in Figure 8) is plotted in Figure 9. The errors are distributed similarly in all states. As expected and found in [21], the random walker yields more accurate results with a statistical mean error of $\approx 1.3^{\circ}$ than the topological watershed with $\approx 4.2^{\circ}$. The results from the random walker agrees well to the error of $1.24^{\circ}$ determined in the more rigorous study and plotted in 3 . The error of the topological watershed is lower than $5.8^{\circ}$, which was determined in [21]. This might be due to larger contact areas which arise from the inherent overlap of the spheres in the numerical simulation and the different distribution of orientations.

In order to investigate the evolution of the individual orientations, a different, scalar value has to be chosen for the comparison. The mean inclination of all orientations is determined at each loading stage of the simulation, because the main changes happen to the vertical component for this particular loading. The inclination of each individual orientation is calculated by:

$\theta=\arccos \left(\frac{z}{\sqrt{x^{2}+y^{2}+z^{2}}}\right)$

The evolution of the mean inclination with respect to the axial strain is plotted in Figure 10. The same trend as qualitatively shown in Figure 8, i.e., an increase in the vertical orientations, can be observed qualitatively using this representation. The evolution of both - the reference and the orientations from the images (ITK and random walker) - follows the same trend with some minor deviations. Upon unloading, the mean inclination partly recovers, i.e., increases, for the reference and the image analysis results.

Although, the individual orientations are strongly biased and do not resemble the reference distribution, the mean inclination of all orientations qualitatively follows and even quantitatively agrees with the reference evolution with deviations not exceeding $0.7^{\circ}$.

Fabric tensors can be used in order to statistically describe the bulk of orientations at a specific state of a granular material [44]. Most commonly, a fabric tensor of 2 nd order is used:

$\mathbf{N}=\frac{1}{C} \sum_{\alpha=1}^{C} \mathbf{o}^{\alpha} \otimes \mathbf{o}^{\alpha}$

with $C$ being the number of contacts and o an individual orientation. Following [44], a deviatoric fabric tensor $\mathbf{D}$ is calculated by:

$\mathbf{D}=\frac{15}{2}\left(\mathbf{N}-\frac{1}{3} \mathbf{I}\right)$

To quantitatively describe anisotropy, a scalar value can be calculated from $\mathbf{D}[45]$ :

$a=\sqrt{\frac{3}{2} \mathbf{D}: \mathbf{D}}$

A fabric tensor is computed at each stage of the simulation for both, the DEM fabric and the orientations determined by image analysis. The evolution of the scalar anisotropy factor $a$ is plotted in Figure 11. As expected from the preparation of the synthetic specimen and the projection plots in Figure 8, the fabric is 


\section{Contact orientations from DEM}
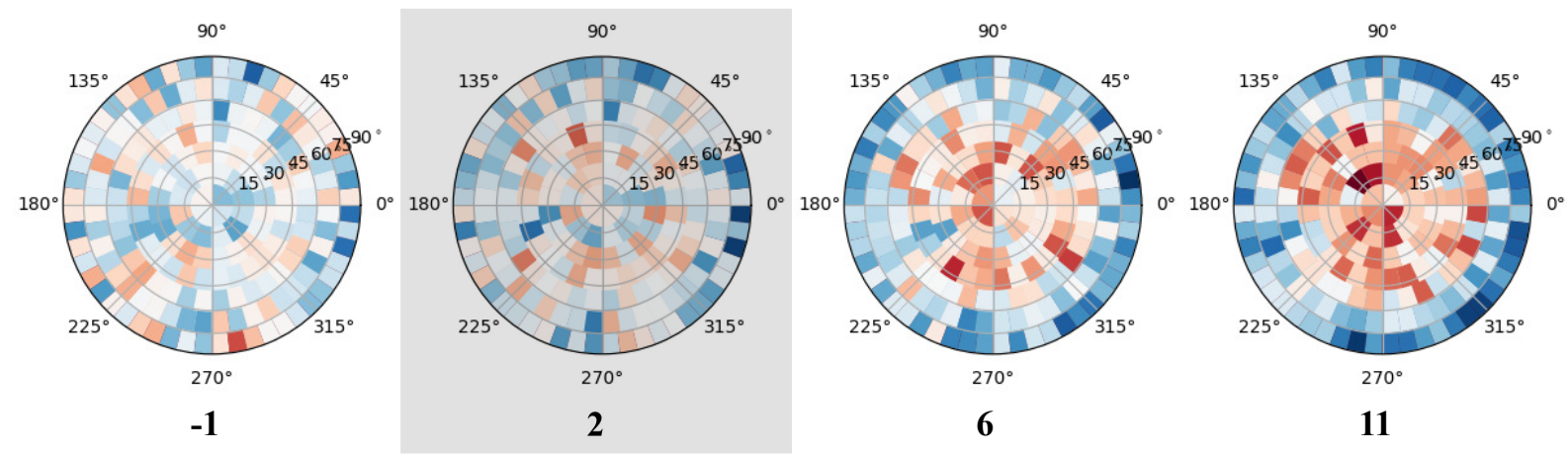

\section{Contact orientations from ITK segmentation}
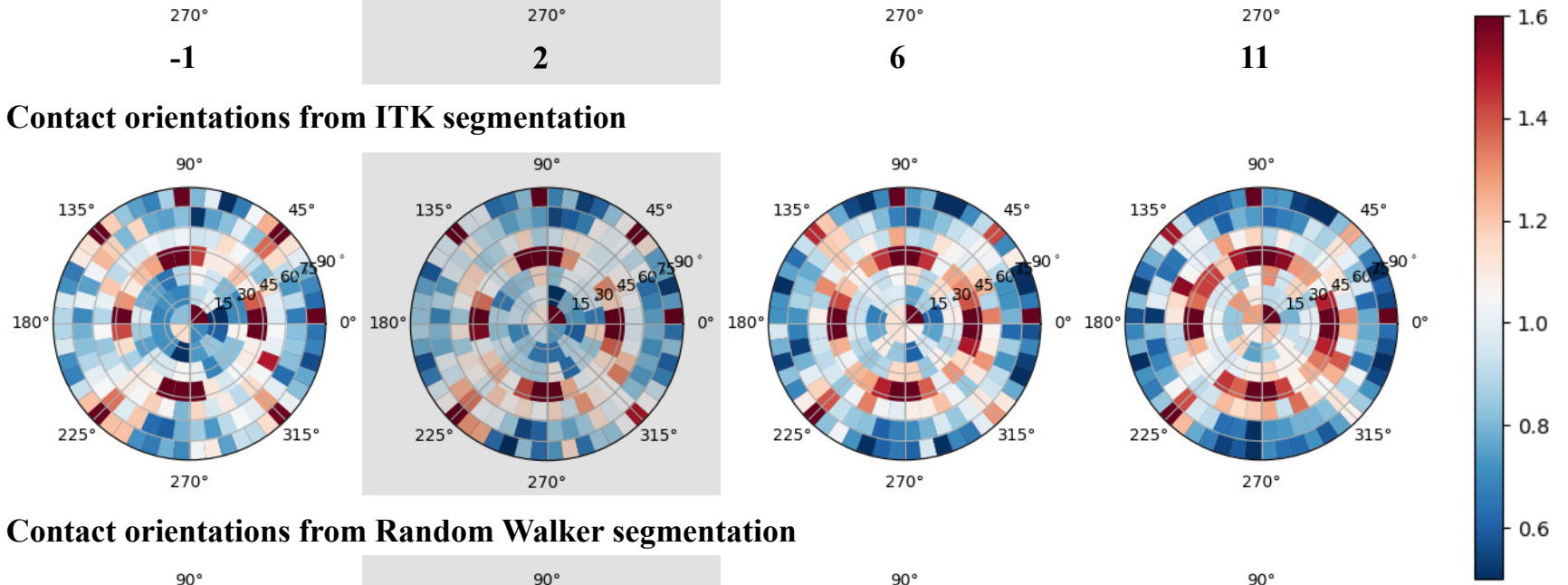

Contact orientations from Random Walker segmentation
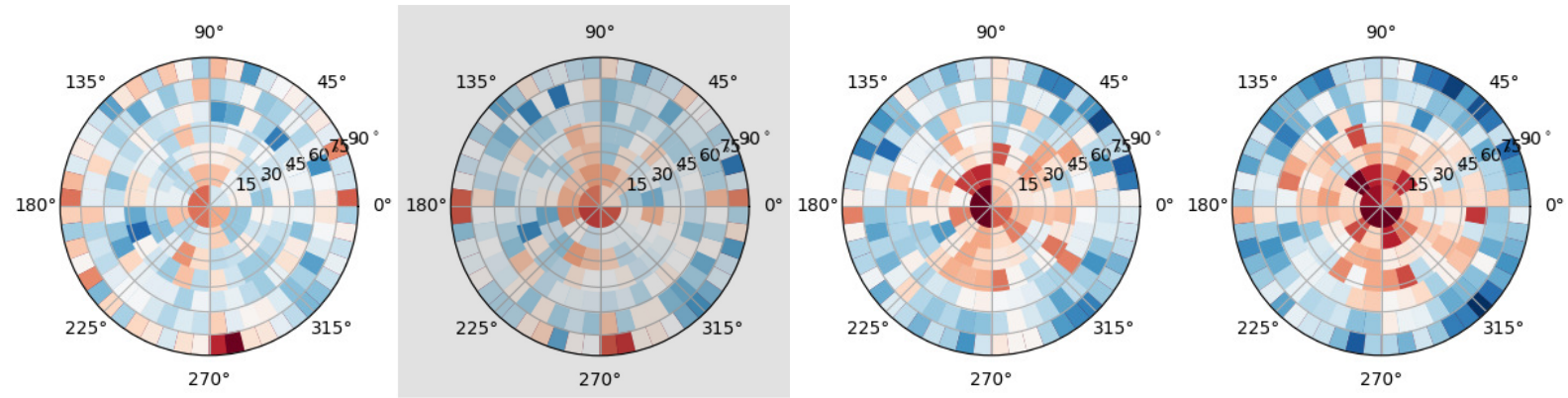

Fig. 8 Binned Lambert azimuthal equal area plots of the contact orientations at different stages of the loading (the labels relate to the macroscopic response in Figure 4) - upper row: orientations directly from the discrete element simulation. middle row: orientations determined from the ITK watershed. lower row: orientations determined with the random walker segmentation. The colors correspond to the normalised density of orientations in the bins - red depicts a high and blue a low density. State 2 is highlighted for a more detailed analysis.

initially isotropic $(a \approx 0)$ with an increasing anisotropy due to the vertical alignment of contacts into the loading direction. The anisotropy then increases as the contact orientations realign with the induced principal stress direction, i.e., the vertical axis.

The results of the random walker and of the ITK watershed are shown in Figure 11. Although the topological watershed yields substantially higher errors and a strong bias when determining individual orientations (see Figures 8 and 9), the fabric anisotropy agrees to the one determined by the random walker. Both evolutions follow the reference fabric qualitatively well and show only minor quantitative deviations.
The reason for the agreement of the anisotropy from the ITK watershed with the random walker as well as the reference is the degree of approximation of the distribution. Even though the orientations from the ITK watershed are strongly biased towards $45^{\circ}$ to the general axes, see Figure 8, the distribution is symmetric to/about the vertical axis. The same holds for the orientations from the random walker and the reference. The orientations from ITK concentrate along the vertical direction with ongoing loading like the orientations from the reference and the random walker. Thus, the anisotropy changes only with the vertical alignment, as the radial distribution remains almost isotropic. 


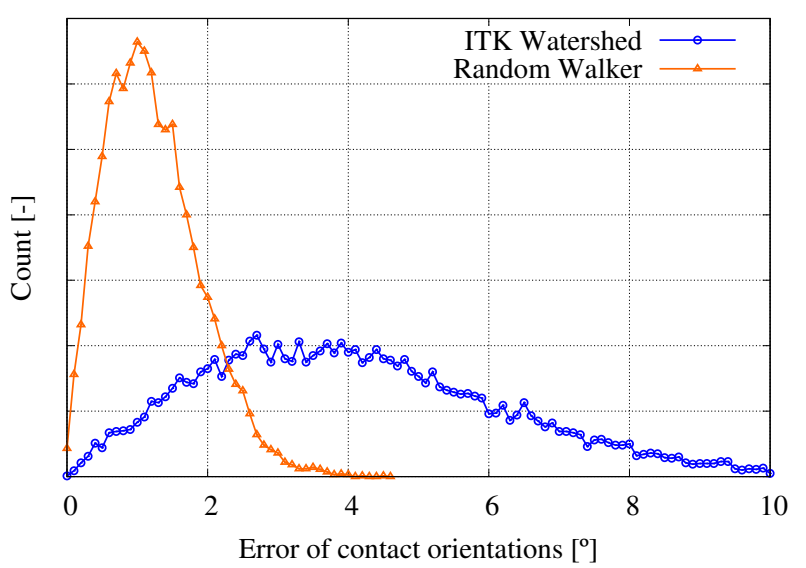

Fig. 9 Histogram of the errors of the individual orientations determined by the ITK watershed and the random walker for state 2 - highlighted in Figure 8 . The errors represent the angles between the orientations from the respective image analysis technique and the reference from the DEM simulation.

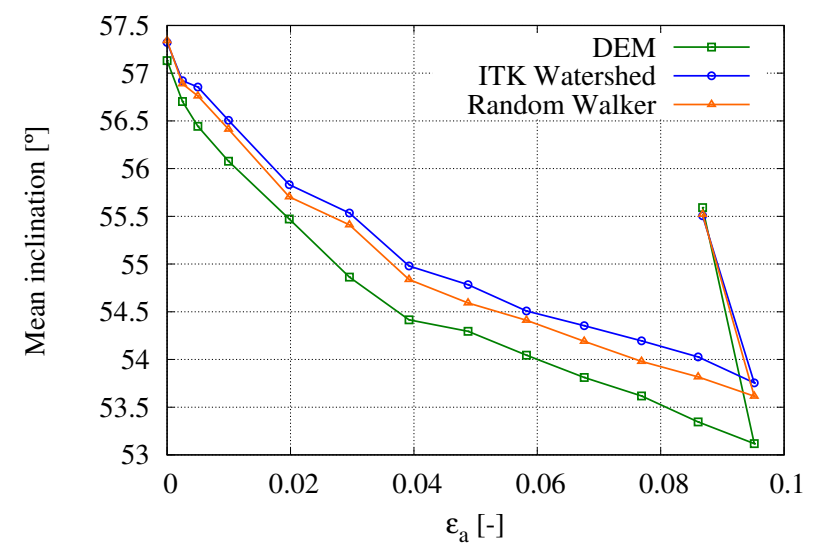

Fig. 10 Plot of the mean inclination of all individual orientations. $0^{\circ}$ being completely vertical and $90^{\circ}$ horizontal. Comparison of the DEM reference and the image analysis results.

We employ a typical 2nd order approximation which is not sufficient to capture the bias from the ITK watershed, because it is radially symmetric. A higher order approximation should be able to show the strong differences of the distributions.

\subsection{Results from a cyclic simulation}

Another question that arises is the sensitivity of the contact fabric and its measurability upon small macroscopic changes, such as unload-reload cycles. In order to investigate this, a DEM simulation similar to the one presented in Figure 4 is carried out. The constitutive parameters are identical and the specimen was prepared similarly. The specimen is unloaded and reloaded seven
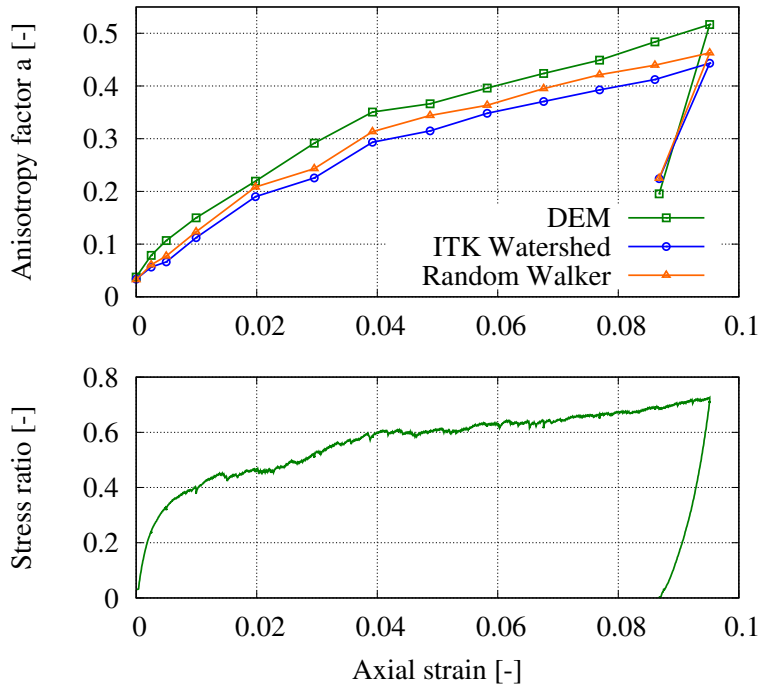

Fig. 11 Evolution of the scalar anisotropy factor with the loading. Comparison of the image analysis results with the reference behaviour from the DEM simulations.

times at different states throughout the loading. Three intermediate states are recorded at every cycle to create the synthetic images and carry out the image analysis that is described above. The macroscopic response of the specimen is shown in Figure 12.

The distribution of contact orientations is again described and compared using the scalar anisotropy factor, see equation (5). Figure 13 shows the evolution of the anisotropy of the reference as well as both image analysis approaches, the ITK watershed and the random walker. Both - the anisotropy from the ITK watershed and the random walker - agree with the reference. On the right side of Figure 13 a zoom-in of the first three cycles is displayed. Both approaches are able to capture even these small changes of the contact anisotropy.

\section{Summary and outlook}

The behaviour of granular materials is known to be governed by the mechanics at the grain-scale. According to [2], the micro-mechanical behaviour may be described by (a) the fabric, (b) the kinematics and (c) the inter-granular forces. A number of contributions have introduced and improved the measurement of continuous and discrete kinematics (b). The measurement of inter-granular forces (c) is still not possible in assemblies of natural granular materials, but recent developments show promising results on small assemblies of synthetic materials $[46,47,48,49]$. Some studies reported on the measurement of granular fabric $[50,17]$, but without studying the metrology of the tools that 

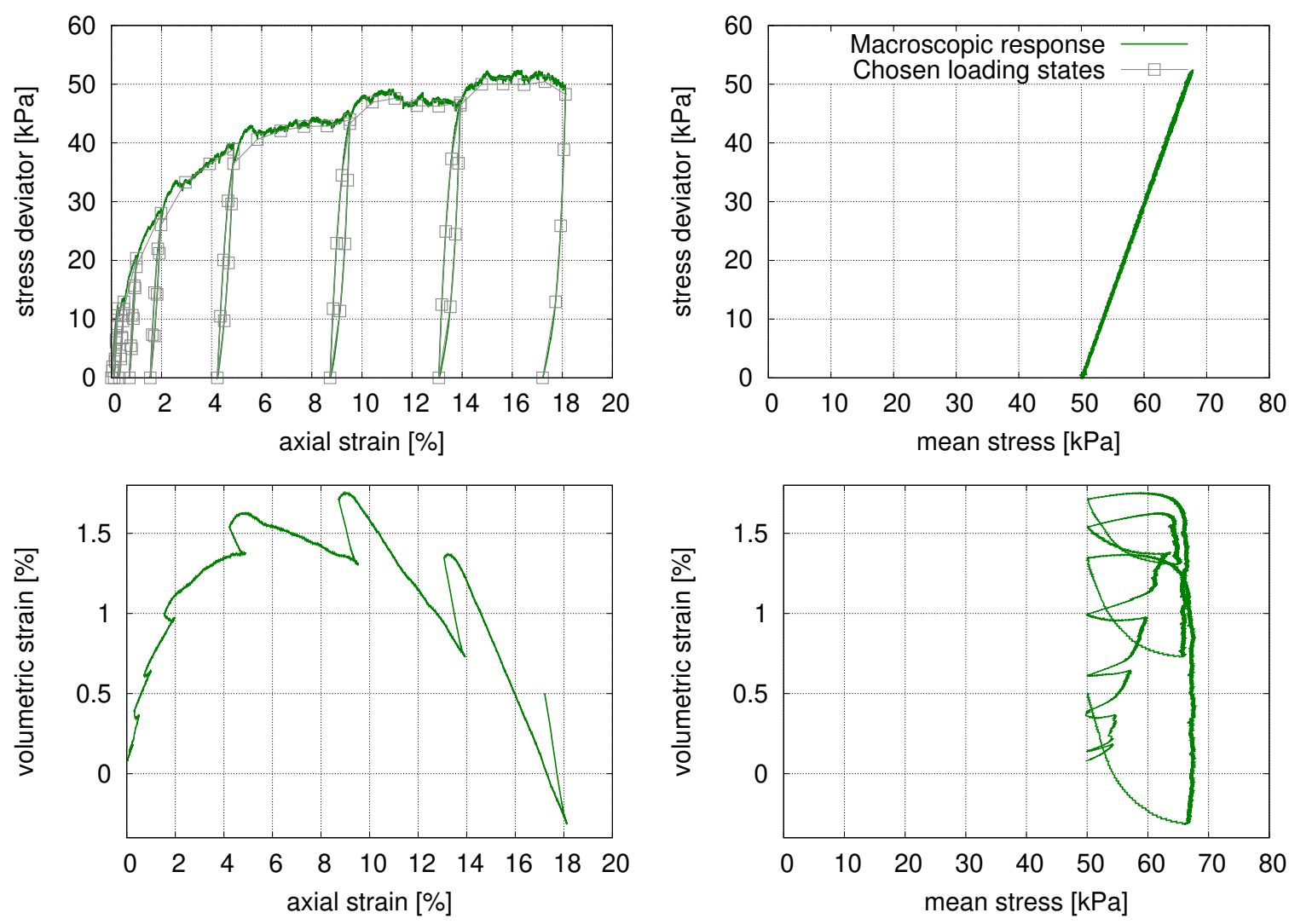

Fig. 12 Macroscopic response of the synthetic specimen to unload / reload cycles.

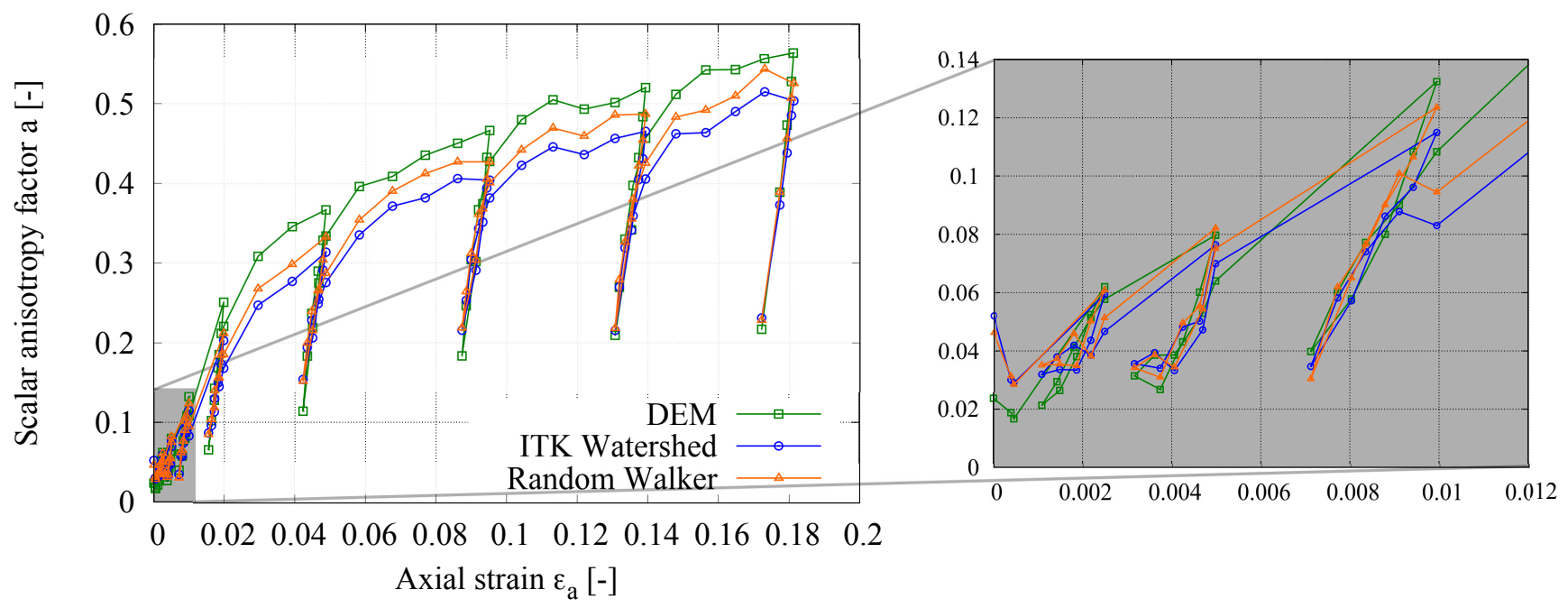

Fig. 13 Evolution of the fabric anisotropy with loading / unloading / reloading cycles. Comparison of the image analysis results with the reference behaviour from the DEM simulations. Right figure: Zoom of the first three cycles.

were used for the measurements. In the present paper, the results of the study on individual contacts [21] have been related and applied to an evolving assembly of thousands of particles.

To achieve that, a discrete element simulation is carried out to provide a reference, i.e., a sequence of perfectly known fabrics at several states of a macroscopic loading. Images of these states are then created using Kalisphera [22], a tool that allows creating images of spheres taking into account the PVE. The other inherent defects, i.e., blur and noise, are applied to obtain realistic and representative synthetic images for the image analysis. The results are compared in terms of detected contacts, their orientations and a second order 
fabric tensor, that is commonly used to statistically express orientational fabric.

In our previous study [21], it was found that contacts are systematically over-detected using standard global thresholding approaches, i.e., particles, that are close to each other but do not touch, are detected to be in contact due to the PVE. This is confirmed for the image of the synthetic assembly by running the global contact detection: $\approx 50 \%$ more contacts are detected in the image than actually present in the reference. The local thresholding approach, that was proposed in [21], is applied to refine the detected contacts and is found to get much closer to the reference, with an over-detection of only $\approx 3 \%$.

The contact orientations calculated on the basis of two different watersheds, i.e., a ITK watershed, which is used for the initial segmentation, and the random walker segmentation as a refinement, are compared either using plots of all individual orientations or a mean inclination (angle with the vertical axis) per analysed state. The orientations from the ITK watershed are strongly biased and only weakly show a tendency to the vertical axis with ongoing loading. On the contrary, the orientations based on the random walker segmentation agree with the reference distribution and show a similar trend/evolution throughout the loading. However, this representation is not suitable for a quantitative comparison and the mean inclination of all orientations is thus chosen as a scalar descriptor. Surprisingly, the evolution of the mean inclination obtained from the ITK watershed agrees to the reference and shows a slight deviation of max. $0.7^{\circ}$. The mean inclination from random walker images follows a similar trend as for the reference; quantitatively, a deviation of $\approx 0.5^{\circ}$ is found.

The orientations can also be statistically captured by a second-order fabric tensor. Comparing the evolution of the anisotropy of the fabric tensors shows a qualitative agreement of both watershed methods with the reference fabric. Quantitatively, the fabrics from the images agree very well with each other, but show a slight deviation from the reference.

The findings of [21] can thus be extended to granular assemblies of spherical particles: a local contact detection is necessary to reduce the over-detection and the random walker segmentation yields qualitatively as well as quantitatively acceptable results. The ITK watershed introduces a strong bias on individual contact orientations, but performs as well as the advanced and more time-consuming random walker segmentation when focused on the evolution of anisotropy for the chosen loading and the order of approximation of the distribution. The latter observation, however, arises due to additional effects, such as the symmetric loading and fabric evolution, as highlighted in the analysis of the results. For different non-symmetric loading situations or a higher order of approximation, the high error and bias of the orientations from the ITK watershed should likely be reflected in the anisotropy as well. Thus, it is still highly recommended to use the random walker segmentation (or other advanced algorithms) to determine contact orientations.

Based on this virtual simulation we expect to be able to capture cyclic behaviour just as well in an x-ray tomography of spherical, and round particles. This will allow inroads into understanding the abrupt stiffness changes observed upon unloading and reloading.

This analysis is restricted to the measurement of contact fabric. A complete description of the geometric fabric of a granular material has also to include other parts, such as particle [4] and void fabric [5] or even combined fabric measures such as presented in [51]. Some of these measures might be extracted more accurately from tomographic images and will be part of future studies.

Acknowledgements We express our thanks to Félix Bertoni for implementing Kalisphera in $\mathrm{C}++$. Laboratoire 3SR is part of the LabEx Tec 21 (Investissements dAvenir - grant agreement $\mathrm{n}^{\circ}$ ANR-11-LABX-0030). We thank the Center for Information Services and High Performance Computing (ZIH) at TU Dresden for generous allocations of computing resources.

\section{Compliance with Ethical Standards}

Conflict of Interest: The authors declare that they have no conflict of interest.

Funding: The research leading to these results has received funding from the German Research Foundation (DFG) $n^{\circ} 254872581$ and from the European Research Council under the European Union's Seventh Framework Program FP7-ERC-IDEAS Advanced Grant Agreement $\mathrm{n}^{\circ} 290963$ (SOMEF).

\section{References}

1. K. Wiendieck. Zur Struktur körniger Medien. Die Bautechnik, 6:196-199, 1967.

2. F. Calvetti, G. Combe, and J. Lanier. Experimental micromechanical analysis of a $2 \mathrm{~d}$ granular material: relation between structure evolution and loading path. Mechanics of Cohesive-frictional Materials, 2(2):121-163, 1997.

3. J. Desrues and G. Viggiani. Strain localization in sand: an overview of the experimental results obtained in Grenoble using stereophotogrammetry. International Journal for Numerical and Analytical Methods in Geomechanics, 28(4):279-321, apr 2004

4. M. Oda. Initial fabrics and their relations to mechanical properties of granular material. Soils and Foundations, $12(1), 1972$. 
5. M. Oda, S. Nemat-Nasser, and J. Konishi. Stress-induced anisotropy in granular masses. Soils and Foundations, 25(3):85-97, 1985.

6. P. A. Cundall and O. D. L. Strack. A discrete numerical model for granular assemblies. Géotechnique, 29(1):47-65, 1979.

7. R. Dobry and T.-T. NG. Discrete modelling of stressstrain behaviour of granular media at small and large strains. Engineering Computations, 9:129-143, 1992.

8. C. O'Sullivan and L. Cui. Micromechanics of granular material response during load reversals: Combined DEM and experimental study. Powder Technology, 193(3):289302 , aug 2009.

9. R. Kawamoto, E. Andò, G. Viggiani, and J. E. Andrade. Level set discrete element method for three-dimensional computations with triaxial case study. Journal of the Mechanics and Physics of Solids, 91:1-13, 2016.

10. J. Desrues, R. Chambon, M. Mokni, and F. Mazerolle. Void ratio evolution inside shear bands in triaxial sand specimens studied by computed tomography. Gotechnique, 46(3):529-546, 1996.

11. S. A. Hall, M. Bornert, and J. Desrues. Discrete and continuum analysis of localised deformation in sand using X-ray $\mu \mathrm{CT}$ and volumetric digital image correlation. Géotechnique, 60(5):315-322, 2010.

12. R. Butterfield, R. M. Harkness, and K. Z. Andrews. A stero-photogrammetric method for measuring displacement fields. Gotechnique, 20(3):308-314, 1970.

13. E. Andò, G. Viggiani, S. A. Hall, and J. Desrues. Experimental micro-mechanics of granular media studied by x-ray tomography: recent results and challenges. Géotechnique Letters, 3:142-146, 2013.

14. E Andò, S. A. Hall, G. Viggiani, J. Desrues, and P. Bésuelle. Grain-scale experimental investigation of localised deformation in sand: a discrete particle tracking approach. Acta Geotechnica, 2012.

15. K. A. Alshibli and B. A. Alramahi. Microscopic evaluation of strain distribution in granular materials during shear. Journal of Geotechnical and Geoenvironmental Engineering, 132(1):80-91, 2006.

16. J. Fonseca, C O'Sullivan, M.R. Coop, and P.D. Lee. Quantifying the evolution of soil fabric during shearing using directional parameters. Géotechnique, 63(6):487499, may 2013.

17. J. Fonseca, S. Nadimi, C.C. Reyes-Aldasoro, C. O'Sullivan, and M.R. Coop. Image-based investigation into the primary fabric of stress-transmitting particles in sand. Soils and Foundations, 56(5):818-834, 2016.

18. A. M. Druckrey, K. A. Alshibli, and R. I. Al-Raoush. 3D characterization of sand particle-to-particle contact and morphology. Computers and Geotechnics, 74:26-35, 2016.

19. V. Cnudde and M. N. Boone. High-resolution X-ray computed tomography in geosciences: A review of the current technology and applications. Earth-Science Reviews, 123:1-17, 2013.

20. S. Weis and M. Schröter. Analyzing X-Ray tomographies of granular packings. Review of Scientific Instruments, (88), 2017.

21. M. Wiebicke, E. Andò, I. Herle, and G. Viggiani. On the metrology of interparticle contacts in sand from x-ray tomography images. Measurement Science and Technology, 28(12):124007, 2017.

22. A. Tengattini and E. Andò. Kalisphera: an analytical tool to reproduce the partial volume effect of spheres imaged in 3D. Measurement Science and Technology, 26(9), 2015.
23. Michael Fox, Tomaso Aste, and Denis Weaire. The Pursuit of Perfect Packing. The Mathematical Gazette, 85(503):370, 2001.

24. Chaoming Song, Ping Wang, and Hernàn A. Makse. A phase diagram for jammed matter. nature letters, 453(May):629-632, 2008.

25. Dapeng Bi, Jie Zhang, Bulbul Chakraborty, and R. P. Behringer. Jamming by shear. Nature, 480(7377):355358, 2011.

26. Adrian Baule and Hernán A. Makse. Fundamental challenges in packing problems: From spherical to nonspherical particles. Soft Matter, 10(25):4423-4429, 2014.

27. G. W Delaney, T. Di Matteo, and T. Aste. Combining tomographic imaging and DEM simulations to investigate the structure of experimental sphere packings. Soft Matter, 6:2992-3006, 2010.

28. T Shire, C O'Sullivan, H Taylor, and W Sim. Measurement of constriction size distributions using three grainscale methods. In Harris, Whitehouse, and Moxon, editors, Proc. 8th International Conference on Scour and Erosion, pages 1067-1073, London, 2016. Taylor \& Francis Group.

29. E. Andò, R. Cailletaud, E. Roubin, O. Stamati, and the spam contributors. spam: The software for the practical analysis of materials. https://ttk.gricad-pages. univ-grenoble-alpes.fr/spam/, 2017-.

30. M. Wiebicke. Benchmark analysis of synthetical images source code and example dem data. http://dx.doi.org/ 10.25532/OPARA-26.

31. P. Iassonov, T. Gebrenegus, and M. Tuller. Segmentation of X-ray computed tomography images of porous materials: A crucial step for characterization and quantitative analysis of pore structures. Water Resources Research, 45(9):1-12, 2009.

32. N. Otsu. A Threshold Selection Method from Gray-Level Histograms. IEEE Transactions on Systems, Man, and Cybernetics, 9:62-66, 1979.

33. F. Meyer and S. Beucher. Morphological segmentation. Journal of Visual Communication and Image Representation, 1(1):21 - 46, 1990.

34. Leo Grady. Random walks for image segmentation. In IEEE Transactions on Pattern Analysis and Machine Intelligence, volume 28, 2006.

35. S. van der Walt, J. L. Schönberger, J. Nunez-Iglesias, F. Boulogne, J. D. Warner, N. Yager, E. Gouillart, T. Yu, and the scikit-image contributors. scikit-image: image processing in Python. PeerJ, 2:e453, 62014.

36. E. Andò. Experimental investigation of microstructural changes in deforming granular media using x-ray tomography. PhD thesis, Université de Grenoble, 2013.

37. T. Aste, M. Saadatfar, and T. J. Senden. Local and global relations between the number of contacts and density in monodisperse sphere packs. Journal of Statistical Mechanics: Theory and Experiment, (7), 2006.

38. T. Aste, M. Saadatfar, and T. J. Senden. Geometrical structure of disordered sphere packings. Physical Review E, 71, 2005.

39. Fabian M. Schaller, Max Neudecker, Mohammad Saadatfar, Gary Delaney, Klaus Mecke, Gerd E. Schröder-Turk, and Matthias Schröter. Tomographic analysis of jammed ellipsoid packings. AIP Conference Proceedings, 1542(June 2013):377-380, 2013.

40. Fabian M. Schaller, Max Neudecker, Mohammad Saadatfar, Gary W. Delaney, Gerd E. Schröder-Turk, and Matthias Schröter. Local origin of global contact numbers in frictional ellipsoid packings. Physical Review Letters, 114(15):1-5, 2015. 
41. V. Šmilauer. Woo Documentation. 2016. https://woodem.org.

42. C. Jaquet, E. Andó, G. Viggiani, and H. Talbot. Estimation of Separating Planes between Touching 3D Objects Using Power Watershed. In International Symposium on Mathematical Morphology, volume 11, 2013.

43. G. Viggiani, E. Andò, C. Jaquet, and H. Talbot. Identifying and following particle-to-particle contacts in real granular media: An experimental challenge. In AIP Conference Proceedings, Powders \& Grains 2013, volume 60, pages $60-65,2013$.

44. Kanatani K.-I. Distribution of directional data and fabric tensors. International Journal of Engineering Science, 22(2):149 - 164, 1984.

45. X. Gu, J. Hu, and M. Huang. Anisotropy of elasticity and fabric of granular soils. Granular Matter, 19(2):1-15, 2017.

46. S. A. Hall, J. Wright, T. Pirling, E. Andò, D. J. Hughes, and G. Viggiani. Can intergranular force transmission be identified in sand? First results of spatially-resolved neutron and X-ray diffraction. Granular Matter, 13(3):251254, 2011.

47. R. Hurley, E. Marteau, G. Ravichandran, and J. E. Andrade. Extracting inter-particle forces in opaque granular materials: Beyond photoelasticity. Journal of the Mechanics and Physics of Solids, 63:154-166, feb 2014.

48. R. C. Hurley, S. A. Hall, J. E. Andrade, and J. Wright. Force measurements in stiff, 3D, opaque granular materials. In EPJ Web of Conferences, Powders ES Grains 2017, volume 140, 2017.

49. W. H. Imseeh and K. A. Alshibli. 3D finite element modelling of force transmission and particle fracture of sand. Computers and Geotechnics, 94(September 2017):184-195, 2018.

50. W. H Imseeh, A. M. Druckrey, and K. A. Alshibli. 3D experimental quantification of fabric and fabric evolution of sheared granular materials using synchrotron microcomputed tomography. Granular Matter, 2018.

51. Matthew R. Kuhn, WaiChing Sun, and Qi Wang. Stressinduced anisotropy in granular materials: fabric, stiffness, and permeability. Acta Geotechnica, 10(4):399-419, 2015. 